Nr. 410 | 24.11.2021

Russland-Analysen

\title{
Erdgashandel
}

ANALYSE

Der deutsch-russische Erdgashandel. Die Ursachen der aktuellen Spannungen

Heiko Pleines (Forschungsstelle Osteuropa an der Universität Bremen)

CHRONIK

Covid-19-Chronik, 01. - 13. November 2021

- CHRONIK

01. - 12. November 2021

Deutsches Polen-Institut
Forschungsstelle Osteuropa an der Universität Bremen
Transformationsökonomien
Leibniz-Institut für Ost- und Südosteuropa-

forschung
Zentrum für Osteuropa- und internationale Studien (ZOiS) gGmbH 


\section{Der deutsch-russische Erdgashandel. Die Ursachen der aktuellen Spannungen}

Heiko Pleines (Forschungsstelle Osteuropa an der Universität Bremen)

DOI: $10.31205 /$ RA.410.01

\section{Zusammenfassung}

Der Import von Erdgas aus Russland ist für Deutschland von großer Bedeutung. Erdgas hat mittlerweile einen Anteil von über einem Viertel am deutschen Primärenergieverbrauch. Importe aus Russland wiederum decken den Erdgasverbrauch mittlerweile etwa zur Hälfte. Die aktuellen außenpolitischen Spannungen und extremen Preisentwicklungen auf dem Erdgasmarkt sind aber nicht durch diese Bedeutung zu erklären. Vielmehr haben sich die Bedingungen des Erdgashandels in den letzten Jahren grundlegend geändert. Zum einen bemüht sich Russland durch den Bau von Pipelines durch internationale Gewässer Transitländer aus dem Handel auszuschließen. Zum anderen hat die EU den europäischen Erdgashandel liberalisiert und damit neue Regeln für Verträge und Preisbildung eingeführt.

\section{Einleitung}

Erdgas hat als Energieträger in Deutschland zunehmend an Bedeutung gewonnen. Sein Anteil am Primärenergieverbrauch hat sich von $15 \%$ im Jahr 1990 mittlerweile fast verdoppelt. Da die eigene deutsche Förderung von Erdgas von geringem Umfang ist und außerdem abnimmt, wird der größte Teil des Erdgasverbrauchs durch Importe gedeckt. Bereits seit 1973 bezieht Deutschland über Pipelines Erdgas aus Russland (damals als Teil der Sowjetunion).

Der Anteil der sowjetischen Erdgaslieferungen an den deutschen Importen stieg bis Ende der 1980er Jahre auf 50 \%. Mit dem Zusammenbruch der Sowjetunion 1991 ging der Anteil der russischen Erdgasexporte leicht zurück (siehe Grafik 1 auf S. 6 im folgenden Statistikteil). Stattdessen stiegen die Anteile von Norwegen und den Niederlanden. 2009 sank der russische Anteil erstmals seit 1983 wieder unter 40 \%. Da die Erdgasvorkommen in der Nordsee mittlerweile zunehmend erschöpft sind, profitierte anschließend vor allem Russland vom Anstieg des deutschen Erdgasverbrauchs. Seit 2017 liefert das Land erstmals über die Hälfte der deutschen Importe (Grafik 2 auf S. 6).

Dieser Anstieg macht allerdings keine zusätzlichen Transportkapazitäten erforderlich. Russland hat nie mehr als $201 \mathrm{Mrd}$. $\mathrm{m}^{3}$ Erdgas pro Jahr nach Europa geliefert (Grafik 3 auf S. 7). Die russischen Lieferkapazitäten betrugen aber schon 2015 ohne Nord Stream 2 insgesamt $288 \mathrm{Mrd} . \mathrm{m}^{3}$. Im nächsten Jahr soll die Kapazität mit Nord Stream 2 und der 2020 in Betrieb genommen TurkStream Pipeline insgesamt 378 Mrd. $\mathrm{m}^{3}$ betragen (Tabelle 1 auf S. 7). Russland könnte seine Erdgasexporte nach Europa also fast verdoppeln. Das ist offensichtlich unrealistisch, da eine Steigerung der Nachfrage in Europa in dieser Form nicht zu erwarten ist.

Russlands aktuelle Energiestrategie, die im April 2020 veröffentlicht wurde, sieht zwar im optimistischen
Szenario eine Verdoppelung der russischen Erdgasexporte bis 2035 vor, aber der Anstieg betrifft vor allem den Export von Flüssiggas per Schiff. Die Erdgasexporte durch Pipelines sollen maximal um ein Drittel steigen und zwar durch Exporte nach China. Russland geht es dementsprechend bei den neuen Pipelineprojekten nach Europa gar nicht um eine Ausweitung der Exportkapazitäten, sondern um eine Änderung der Transportwege.

\section{Transportwege}

Das sowjetische Pipelinesystem lieferte Erdgas aus Westsibirien durch die Belarussische und Ukrainische Sowjetrepublik in die sozialistischen Staaten Mittelosteuropas. Von dort wurden die Exporte ausgeweitet über Deutschland und Österreich nach Westeuropa sowie über Rumänien auf den Balkan. Diese Pipelines, mit einer Gesamtkapazität von $183 \mathrm{Mrd}$. $\mathrm{m}^{3}$, liefen alle über das Territorium der Ukraine, so dass die Ukraine nach dem Zerfall der Sowjetunion ein Monopol auf den Transit russischer Erdgasexporte hatte. Gleichzeitig war die Ukraine auch ein großer Abnehmer russischen Erdgases.

Erdgas wurde zu einem zentralen Streitpunkt zwischen Russland und der Ukraine. Diese Konflikte führten bereits seit den 1990er Jahren zu russischen Lieferstopps für die Ukraine. Als die Ukraine darauf 2006 und 2009 mit der Entnahme von für den Transit bestimmten Erdgases reagierte, um den Eigenbedarf zu decken, kam es auch zu einer vorübergehenden Einstellung der russischen Lieferungen in die EU.

Da einige der östlichen Mitgliedsstaaten der EU in ihrer Erdgasversorgung vollständig von Russland abhängen, führte der Lieferausfall vor allem im Januar 2009 zu akuten Problemen. Die EU reagierte darauf, indem sie die von Ost nach West verlaufenden Erdgaspipelines so aufrüstete, dass die Fließrichtung umgekehrt werden kann. Dementsprechend kann bei einem erneuten 
Lieferausfall Erdgas von Deutschland oder Österreich nach Osten geliefert werden. Diese umgekehrte Fließrichtung wird auch bereits seit 2016 genutzt, um die Ukraine vor allem aus der Slowakei mit Erdgas zu versorgen. (Die Bedeutung des russischen Erdgastransits für die Ukraine wird in den Ukraine-Analysen Nr. 258 ausführlicher betrachtet.)

Russland reagierte auf die Erdgaskrisen mit der Ukraine mit dem erklärten Ziel, die Ukraine als Transitland auszuschalten. Die ersten post-sowjetischen Pipelineprojekte Russlands, die Jamal-Pipeline (1999) durch Belarus und Polen sowie die Blue Stream Pipeline (2005) durch das Schwarze Meer in die Türkei dienten zwar vorrangig der Expansion der Exportkapazitäten nach Westeuropa und der Erschließung des türkischen Marktes, sie umgingen aber schon bewusst die Ukraine. Die folgenden Projekte, Nord Stream 1 und 2 (2011 bzw. 2021) durch die Ostsee sowie TurkStream (2020) durch das Schwarze Meer, sollten den Erdgastransit durch die Ukraine weitgehend überflüssig machen. Dies wurde sowohl von Vertretern der russischen Regierung als auch Vertretern des russischen, staatlich kontrollierten Erdgaskonzerns Gazprom mehrfach explizit erklärt. Mit dem Bau der Pipeline durch internationale Gewässer konnten auch weitere Transitländer umgangen werden.

Wie wichtig dieses Ziel genommen wird, zeigt sich auch darin, dass sich Russland trotz massiven Drucks von Seiten der EU 2019 geweigert hat, mit der Ukraine einen Transitvertrag abzuschließen, der über das Jahr 2024 hinausgeht. Bereits jetzt ist der Erdgastransit durch die Ukraine auf ein Minimum reduziert worden. Selbst die gestiegene europäische Nachfrage im Herbst 2021 wurde dementsprechend von russischer Seite nicht mit Hilfe zusätzlicher Lieferungen durch die Ukraine gedeckt. Aus russischer Sicht war es nur logisch, die Inbetriebnahme von Nord Stream 2 als Lösung für erhöhte Gaslieferungen nach Deutschland zu präsentieren.

In Zukunft könnte Gazprom seine in Deutschland erworbenen Gasspeicherkapazitäten nutzen, um auf kurzfristige Nachfragespitzen zu reagieren. Die deutschen Speicher können Erdgas im Umfang von einem Viertel des Jahresverbrauchs aufnehmen. Gazprom kontrolliert fast ein Drittel der Gasspeicher in Deutschland, Österreich und den Niederlanden.

\section{Interessen}

Das russische Interesse an der Vermeidung von Transitländern hat einen wirtschaftlichen Hintergrund. Transitgebühren werden gespart und das Risiko von Lieferausfällen wird reduziert. Im Fall der Ukraine kommt aber verstärkt seit der Ukraine-Krise 2014 ein geopolitisches Motiv hinzu, da die Ukraine - oder zumindest die ukrainische Regierung - als Gegner wahrgenommen wird.
Dieses geopolitische Motiv beunruhigt die Transitländer, insbesondere die Ukraine und Polen. Dabei geht es nicht nur um den Wegfall von Transiteinnahmen, sondern auch um den Verlust einer strategischen Absicherung gegen russischen Druck.

Genau dieses geopolitische Motiv ist auch die Ursache für die amerikanischen Sanktionen gegen Nord Stream 2, denn ohne Nord Stream 2 muss der entsprechende Transit durch die Ukraine erfolgen. Die in deutschen Medien oft geäußerte Behauptung, die USA wollten ihr eigenes Flüssiggas nach Deutschland verkaufen, macht hingegen keinen Sinn. Denn es geht ja gar nicht um eine Reduzierung russischer Erdgaslieferungen, sondern nur um den Transportweg. Wenn das Erdgas nicht durch die Ostsee, sondern durch die Ukraine nach Deutschland kommt, ergibt sich keine zusätzliche Nachfrage für amerikanische Lieferungen.

Für die europäischen Energieunternehmen, die ihr Erdgas aus Russland beziehen, ist hingegen eine gute Beziehung zum Lieferanten zentral. Dementsprechend unterstützen sie die russischen Projekte. Die Bundesregierung hat sich hier eindeutig hinter die Interessen der deutschen Energieunternehmen gestellt. Das im Kompromiss mit den USA zur Umsetzung von Nord Stream 2 vorgesehene Versprechen, sich »zu bemühen«, dass das russisch-ukrainische Transitabkommen um weitere zehn Jahre verlängert wird, dürfte in Anbetracht der klaren russischen Haltung bedeutungslos sein.

\section{Liberalisierung des europäischen Erdgasmarktes}

Die Lieferverträge im russischen Erdgashandel hatten traditionell eine Laufzeit von mehreren Jahrzehnten und sahen eine jährliche Mindestabnahme vor, die auch bezahlt werden musste, wenn sie tatsächlich nicht bezogen wurde. Diese Bedingungen sollten die Rentabilität der Pipelineprojekte sicherstellen. Der Bau einer Erdgaspipeline kostet extrem hohe Summen, im Fall von Nord Stream 2 zum Beispiel mindestens $8 \mathrm{Mrd}$. Euro. Solche Investitionen lohnen sich nur, wenn die langfristige Nutzung der Pipeline garantiert ist. Die Lieferverträge des deutschen Abnehmers E.ON Ruhrgas mit Gazprom hatten so bereits 2008 eine Laufzeit bis 2036.

Da Erdgas ursprünglich als Konkurrent zu Erdöl antrat, wurde im Interesse der Abnehmer der Lieferpreis für Erdgas über komplexe Formeln an den Preis für Erdöl gekoppelt. Im Falle sinkender Erdölpreise sank damit auch der Preis der Erdgasimporte, so dass Erdgas auf dem Importmarkt konkurrenzfähig blieb. Zusätzlich sahen die Lieferverträge, offensichtlich im Interesse Russlands, ein Verbot des Weiterverkaufs an andere Großhändler vor. Die Verträge unterlagen der Geheimhaltung. Dementsprechend hatte Russland mit jedem einzelnen Energieunternehmen eigene, von der Konkur- 
renz isolierte Vertragsbeziehungen. Dies passte auch zu den regionalen Monopolen deutscher Energieversorger.

Solche Märkte, in denen die Beziehungen zwischen Produzenten und Abnehmern von teurer Transportinfrastruktur abhängen, wurden in der Wirtschaftswissenschaft als »natürliche Monopole« bezeichnet. Ein Unternehmen, das diese teure Infrastruktur einmal gebaut hatte, konnte diese nutzen, um alle Kunden, die an diese Infrastruktur angebunden waren, exklusiv zu beliefern. Für die Konkurrenz macht es keinen Sinn einen parallelen zweiten Transportweg zu bauen. So entsteht "natürlich" ein Monopol des Unternehmens, welches als erstes den Kunden erreicht.

In den 1990er Jahren begann die Wirtschaftspolitik in vielen Ländern, die entsprechenden Märkte zu liberalisieren. Zentral dafür war die Trennung von Produktion und Transport. Im Extrem wurde eine strikte Trennung erwartet, so dass ein Produzent seine Transportinfrastruktur verkaufen musste. Als Kompromiss wurden Unternehmen gezwungen, der Konkurrenz gleichberechtigten Zugang zur Transportinfrastruktur zu gewähren. Gleichzeitig wurden die Rechte der Kunden bei Vertragskündigungen und Anbieterwechseln gestärkt. Nach diesem Muster wurden in der EU die Telekommunikation (1998), der Strommarkt (1998) oder die Eisenbahn (Güter 2007, Personen 2020) liberalisiert.

Die entscheidenden Regeln für die Liberalisierung des Erdgasmarktes wurden 2009 verabschiedet. Die Vorgaben für den Zugang von Drittanbietern zu Pipelinekapazitäten traten erst 2015 in Kraft und erfassten nicht bereits bestehende Lieferverträge. Die Trennung von Produktion und Transport betrifft auch Erdgasspeicher, so dass Gazprom als Produzent der Konkurrenz fairen Zugang zu eigener Speicherkapazität innerhalb der EU gewährleisten muss.

Die Regeln beziehen sich nur auf den EU-Binnenmarkt. Ursprünglich betrafen sie deshalb keine Pipelines, die nur bis zur Grenze des Binnenmarktes liefern. Die russische Exportpipeline Nord Stream 1, die von Russland durch die Ostsee nach Deutschland verläuft, war also nicht betroffen. Hier konnte Gazprom die volle Kapazität für eigene Lieferungen nutzen. Die Anschlusspipelines, die von der deutschen Ostseeküste bei Greifswald das Erdgas weitertransportieren sollten, fielen aber unter die Regeln, so dass Gazprom hier die volle Kapazität nicht nutzen konnte. Da der Weitertransport deshalb nicht in vollem Umfang gewährleistet war, blieben die ursprünglichen Lieferungen durch Nord Stream 1 auch unterhalb der Kapazität.

In Reaktion auf Nord Stream 2 einigte sich die EU 2019 darauf, dass die Binnenmarktregeln auch in den Hoheitsgewässern von EU-Mitgliedsstaaten gelten, so dass die deutsche Regulierungsbehörde, die Bundesnetzagentur, für den Schlussabschnitt von Nord Stream 2, und damit de facto für die Lieferkapazitäten der gesamten Pipeline, zuständig ist.

Da Gazprom seine Geschäfte nicht sofort an die neuen EU-Regeln anpasste, geriet es - wie z. B. auch aktuell amerikanische Internet-Unternehmen - in Konflikt mit EU-Recht. Die EU Kommission eröffnete so 2012 ein Kartellverfahren gegen Gazprom. Ein polnisches Gericht nutzte den Vorwurf der marktbeherrschenden Vormachtstellung, um ein Urteil gegen die geplante Nord Stream 2-Pipeline zu verhängen. In Reaktion auf dieses Urteil mussten sich europäische Energieunternehmen aus dem Pipeline-Konsortium zurückziehen.

\section{Interessen}

Die viele Branchen und mächtige Wirtschaftsinteressen betreffende Liberalisierung, die bereits Anfang der 1990er Jahre eingeleitet wurde, ist ein zentrales Ziel der EU. Im Falle des Erdgasmarktes wurde die Liberalisierung aber auch von Akteuren genutzt, die weniger den Wettbewerb als die Geopolitik im Blick haben. Dies hat sich insbesondere beim Konflikt um Nord Stream 2 gezeigt.

Die Liberalisierung, das heißt die Abkehr von langfristigen Preisbindungen und dem Verbot des Weiterkaufs, hat aber auch europäischen Energieunternehmen Vorteile gebracht. RWE erreichte so über ein Schiedsgerichtsurteil 2013 eine Aufhebung der Ölpreisbindung in den Lieferverträgen und erhielt von Gazprom Schadensersatz von mehr als einer Milliarde Euro für seit 2010 über dem Marktniveau gezahlte Preise. Hintergrund war der Bedeutungsgewinn von Flüssiggas, das per Schiff transportiert werden kann und ähnlich wie Erdöl bei Lieferung auf Spotmärkten, also kurzfristig an den Meistbietenden, verkauft wird. Flüssiggas konnte so zu Beginn der 2010er Jahre in Rotterdam unterhalb der von Gazprom gesetzten Preise gekauft werden und über niederländische Pipelines nach Deutschland transportiert werden.

Gazprom als Verkäufer hat logischerweise kein Interesse an niedrigen Preisen. Russland reagierte auf das Risiko stärker schwankender und vor allem fallender Preise bereits 2008 mit Plänen für eine Gas-OPEC. Die Idee dahinter war, ähnlich wie bei der OPEC selber, dass Absprachen der größten Produzenten die Weltmarktpreise im eigenen Interesse beeinflussen können. So wurde mit Katar auch der größte Exporteur von Flüssiggas in die Gas-OPEC einbezogen. Das im selben Jahr in Russland gegründete Forum Gas exportierender Länder (Gas Exporting Countries Forum - GECF), wurde zwar von Russland als Gas-OPEC bezeichnet, konnte sich aber nicht auf eine Einflussnahme auf Fördermengen oder Preisgestaltung einigen.

Es ist offensichtlich, dass das russische Interesse in einer Maximierung der eigenen Einnahmen aus dem 
Erdgasexport besteht und nicht in einer möglichst günstigen und sicheren Versorgung der Abnehmerländer. Dementsprechend sah Russland auch keinen Grund im Herbst 2021 mehr als die vereinbarten Mengen an Erdgas nach Europa zu liefern, um zu fallenden Preisen beizutragen. Die globalen Verwerfungen infolge der Corona-Pandemie haben vielmehr dazu geführt, dass Russland vorübergehend alleine erreichen konnte, was ihm die Gas-OPEC schon 2008 bringen sollte: steigende Preise durch ein reduziertes Angebot. Während vor zehn Jahren Gazproms Kunden die Vorteile der fehlenden Preisbindung nutzten, profitiert jetzt das russische Unternehmen selber.

\section{Ausblick}

Längerfristige Prognosen für die Entwicklung des Erdgasmarktes haben sich in der Vergangenheit regelmäßig als völlig falsch erwiesen. Das Zusammenspiel von Angebot und Nachfrage ist zu komplex. Auf der Angebotsseite gibt es zum Beispiel viele Produzenten und die Frage, wie schnell und in welchem Umfang Terminals für den Transport von Flüssiggas fertiggestellt werden. Die Nachfrage wiederum hängt u. a. vom durch die Corona-Krise stärker als sonst schwankenden Wirtschaftswachstum sowie von den Plänen für die Energiewende $a b$, bei der Erdgas als fossiler Brennstoff an Bedeutung verlieren oder wegen seines vergleichsweise geringen $\mathrm{CO}_{2}$-Gehalts in einer Übergangsphase vermehrt zum Einsatz kommen kann.

Konkret für den russisch-deutschen Erdgashandel ist festzustellen, dass ehrgeizige russische Produktionsziele nicht immer erreicht wurden. Gleichzeitig ist der
Bau von Anlagen für die Verschiffung von Flüssiggas in Russland noch im Anfangsstadium und in Deutschland sogar nur im Planungsstadium. Der Anteil von Erdgas am deutschen Energiemix hängt auch von politischen Entscheidungen im Rahmen der Energiewende ab, die gerade neu verhandelt werden.

Festzuhalten ist aber, dass Russland derzeit keine Pläne hat, seine Exporte durch Erdgaspipelines nach Europa auszuweiten. Für Deutschland bedeutet dies, dass der Erdgashandel mit Russland nur begrenztes Wachstumspotential hat. Falls der deutsche Gesamtverbrauch an Erdgas bis Ende des Jahrzehnts zurückgeht, dürfte aber der russische Anteil an den deutschen Erdgasimporten steigen, da russische Lieferungen konstant bleiben werden, während die Niederlande bis dahin ihre Erdgaslieferungen komplett einstellen werden.

Für eine Ausweitung seiner Exporte setzt Russland auf Flüssiggas. Da im Zusammenhang mit der Energiewende der Verbrauch von Erdgas noch schwerer vorherzusagen ist als bisher, ist diese Flexibilität wirtschaftlich sinnvoll, solange Erdgas trotz der Klimafolgen längerfristig genutzt wird. Falls Deutschland in Zukunft Flüssiggas von Russland beziehen sollte, nachdem beide Länder die entsprechende Infrastruktur geschaffen haben, ergibt sich daraus keine größere Abhängigkeit, da hier Lieferanten - genau wie aktuell beim Erdöl - kurzfristig gewechselt werden können. Da die Zahl der Produzenten von Flüssiggas derzeit zunimmt und die USA und Australien zu den größten Exporteuren von Flüssiggas gehören, ist es schwer vorstellbar, dass doch noch eine Gas-OPEC entsteht. Entscheidend für die Bedeutung von Flüssiggas dürfte vielmehr die Klimapolitik sein.

\section{Über den Autor}

Prof. Dr. Heiko Pleines leitet die Abteilung Politik und Wirtschaft der Forschungsstelle Osteuropa an der Universität Bremen. Er beschäftigt sich seit den 1990er Jahren mit der Energiewirtschaft. Er ist Mitglied der internationalen Studiengruppe »Energy Materiality: Infrastructure, Spatiality and Power« am Hanse-Wissenschaftskolleg Delmenhorst.

\section{Lesetipps:}

- Götz, Roland. (2020): Russlands Energiestrategie bis zum Jahr 2035: Business as usual. In: Russland-Analysen 386, 8 - 12, online: https://www.laender-analysen.de/russland-analysen/386/russland-energiestrategie-2035/.

- Kardas, Szymon; Kononczuk, Wojciech (2020): Vorübergehende Stabilisierung: Der russisch-ukrainische Vertrag zum Gastransit. In: Russland-Analysen 380, 2 - 5, https://www.laender-analysen.de/russland-analysen/380/ russisch-ukrainischer-vertrag-zum-gastransit-2019\%.

- Kusznir, Julia (2019): Nord Stream 2: (Wie) weiter? In: Russland-Analysen 379, 14 - 15, https://www.laender-analysen. de/russland-analysen/379/nord-stream-2-wie-weiter/. 


\section{Der russisch-deutsche Erdgashandel}

Grafik 1: Anteil der Sowjetunion / Russlands an den deutschen Erdgasimporten 1973 - 2002 (in \%)

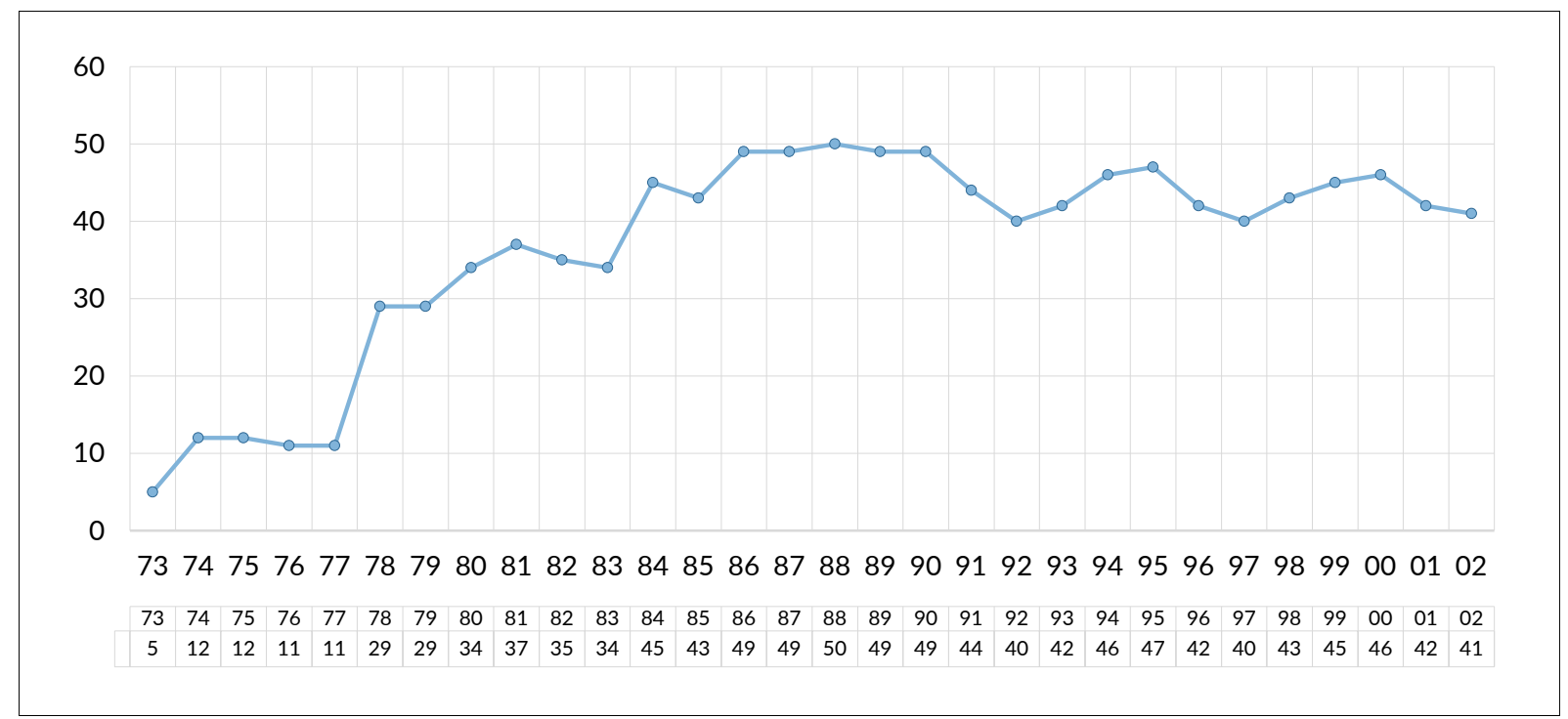

Quelle: Bundesamt für Wirtschaft und Ausfuhrkontrolle, https://www.bafa.de/DE/Energie/Rohstoffe/Erdgasstatistik/erdgas_node.html, Zahlen beziehen sich auf den Brennwert, eigene Berechnung der Prozentanteile

\section{Grafik 2: Anteil Russlands an den deutschen Erdgasimporten 2007 - 2020 (in \%)}

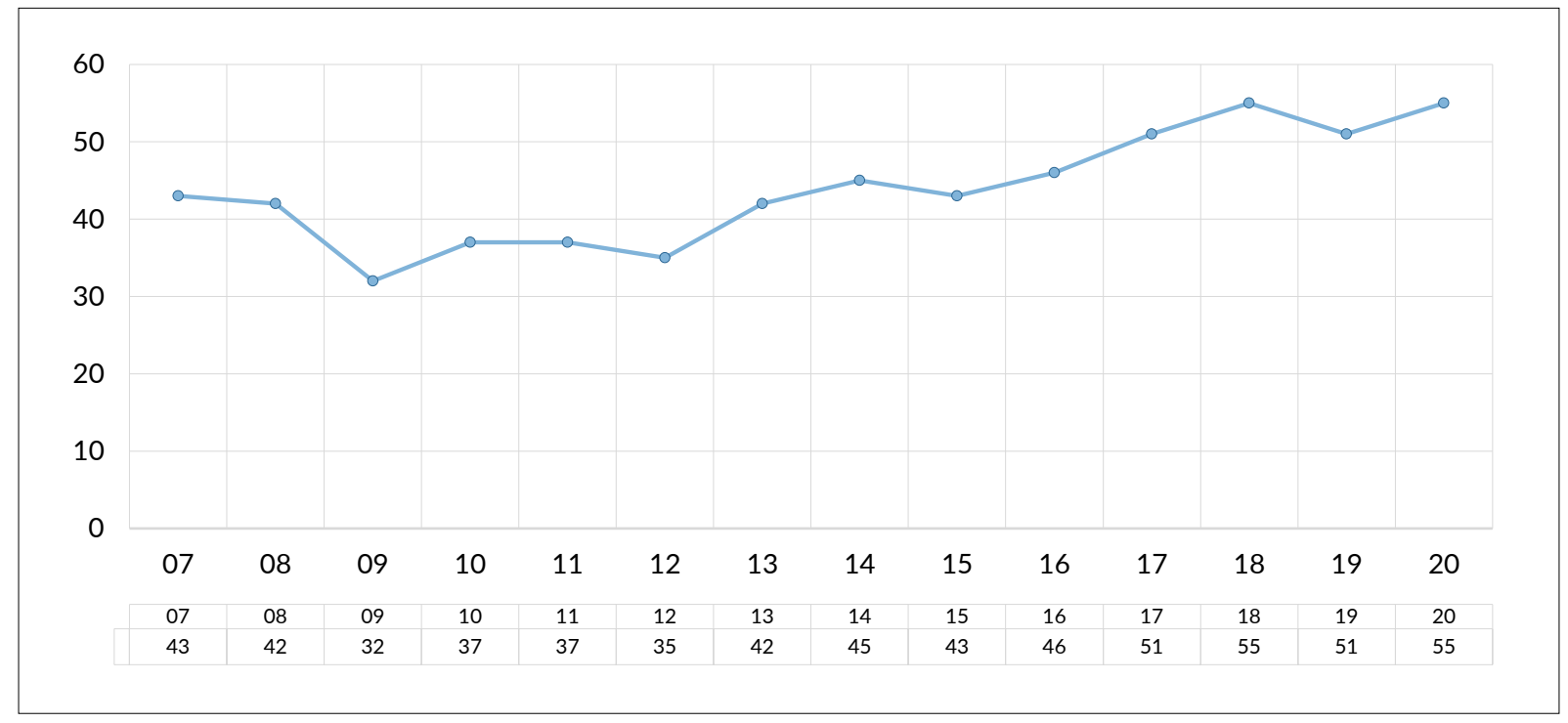

Quelle: BP Statistical Review of World Energy, entsprechende Jahre, Zahlen beziehen sich auf das Volumen, eigene Berechnung der Prozentanteile aus der Tabelle »Trade movements by pipeline" 
Grafik 3: Russische Erdgasexporte nach Europa (einschließlich Türkei, ohne ehemalige Sowjetunion) in Mrd. $\mathrm{m}^{3}$

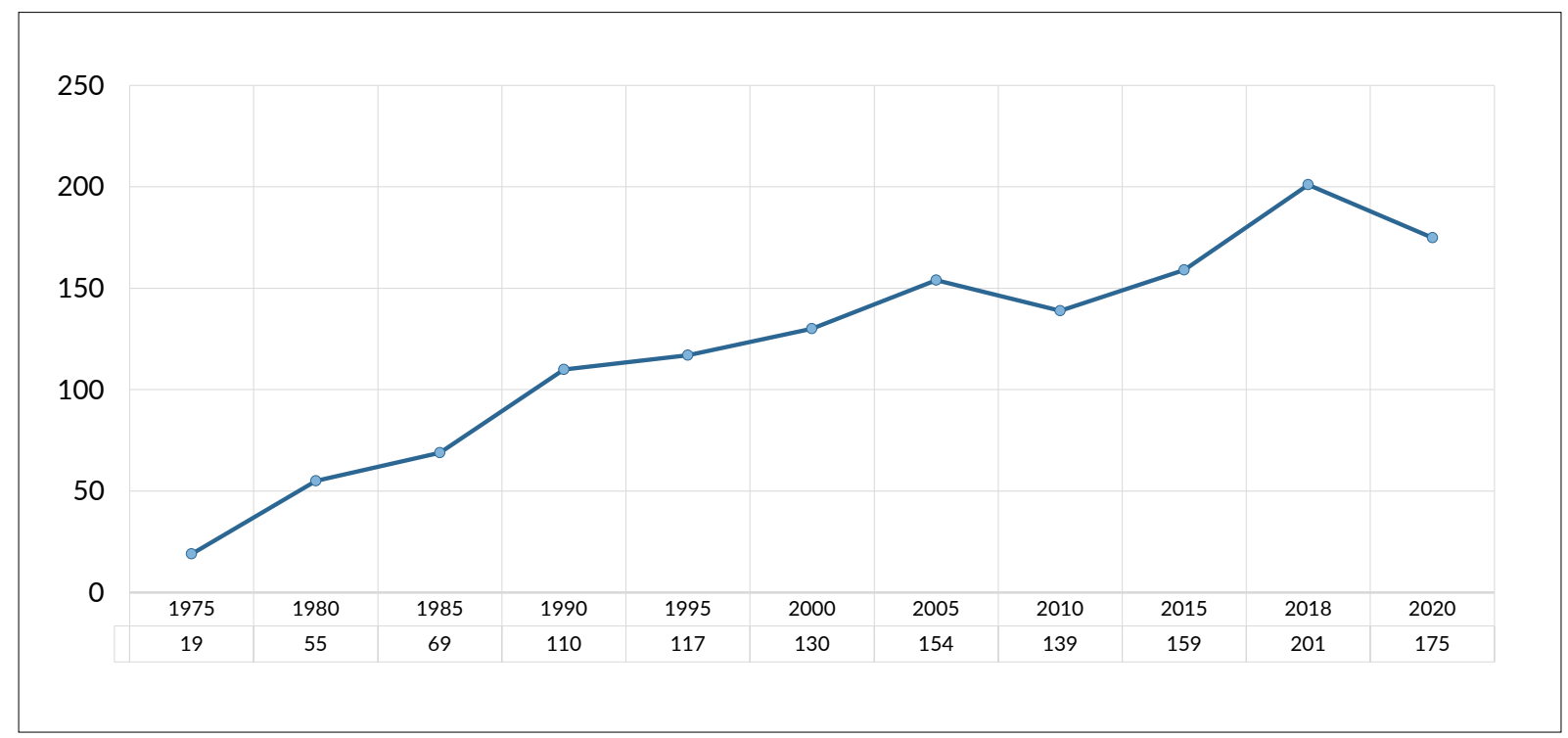

Quelle: Unternehmensangaben von Gazpromexport, http://www.gazpromexport.ru/en/statistics/

Tabelle 1: Pipeline-Optionen für russische Erdgasexporte nach Europa

\begin{tabular}{|c|c|c|c|}
\hline Pipelines & Route & $\begin{array}{l}\text { Kapazität } 2015 \\
\quad\left(\text { Mrd. } \mathrm{m}^{3}\right)\end{array}$ & $\begin{array}{c}\text { Kapazität } 2022 \\
\text { (geplant, Mrd. } \text { m³) }^{3}\end{array}$ \\
\hline $\begin{array}{l}\text { Bruderschaft (sowjetisches } \\
\text { Pipelinenetz) }\end{array}$ & Russland-Ukraine-Mitteleuropa & 100 & 100 \\
\hline $\begin{array}{l}\text { Nordlicht (sowjetisches } \\
\text { Pipelinenetz) }\end{array}$ & Russland-Belarus-Ukraine-Mitteleuropa & 55 & 55 \\
\hline $\begin{array}{l}\text { Trans-Balkan (sowjetisches } \\
\text { Pipelinenetz) }\end{array}$ & Russland-Ukraine-Balkan-Türkei & 28 & 28 \\
\hline Jamal-Europa (seit 1999) & Russland-Belarus-Polen-Westeuropa & 34 & 34 \\
\hline Blue Stream (seit 2005) & Russland-Schwarzes Meer-Türkei & 16 & 19 \\
\hline Nord Stream (seit 2011) & Russland-Ostsee-Deutschland & 55 & 110 \\
\hline TurkStream (seit 2020) & Russland-Schwarzes Meer-Türkei & - & 32 \\
\hline Gesamtkapazität & & 288 & 378 \\
\hline
\end{tabular}

Anmerkung: Die direkte Exportpipeline nach Finnland, über die 2020 nur 1,6 Mrd. $\mathrm{m}^{3}$ Erdgas geliefert wurden, wurde nicht aufgenommen. Quelle: eigene Zusammenstellung (Forschungsstelle Osteuropa an der Universität Bremen) 
Tabelle 2: $\quad$ Schritte vor der Inbetriebnahme von Nord Stream 2 (Stand: 16. November 2021)

\begin{tabular}{|c|c|}
\hline Staatliche Überprüfung und Freigabe & Gerichtsverfahren \\
\hline $\begin{array}{l}\text { - Technische Überprüfung - Bergamt Stralsund: erfolgt } \\
\text { - Zertifizierung (Trennung von Produktion und Transport) - } \\
\text { Bundesnetzagentur: ursprünglich bis Januar } 2022 \text { - aus } \\
\text { formalen Gründen am 16.11.2021 ausgesetzt, bis die } \\
\text { Betreiberfirma »in einer Rechtsform nach deutschem Recht } \\
\text { organisiert ist». } \\
\text { - Überprüfung gemäß EU-Erdgasrichtlinie - Europäische } \\
\text { Kommission: innerhalb von } 4 \text { Monaten nach der Zertifizie- } \\
\text { rung } \\
\text { - Freigabe durch Bundesnetzagentur: innerhalb von } 2 \\
\text { Monaten nach der Überprüfung }\end{array}$ & $\begin{array}{l}\text { - Klage der Nord Stream } 2 \text { AG vor dem Bundes- } \\
\text { gerichtshof und dem Europäischen Gerichtshof gegen } \\
\text { die Anwendung der } 2019 \text { beschlossenen Ausweitung } \\
\text { der Zuständigkeit der Bundesnetzagentur } \\
\text { - Klage des Naturschutzbund Deutschland beim Ver- } \\
\text { waltungsgericht Hamburg gegen das Bundesamt } \\
\text { für Seeschifffahrt und Hydrographie bezüglich der } \\
\text { Genehmigung von Nord Stream } 2 \text { (Eine entsprechen- } \\
\text { de Klage der Deutschen Umwelthilfe gegen das Berg- } \\
\text { amt Stralsund wurde vom Oberverwaltungsgericht } \\
\text { Greifswald am 16.11.2021 abgelehnt.) }\end{array}$ \\
\hline
\end{tabular}

Quelle: eigene Zusammenstellung nach einem Bericht der Deutschen Presse-Agentur, siehe auch https://www.rnd.de/politik/nord-stream-2-welchehuerden-stehen-jetzt-vor-inbetriebnahme-der-ostsee-pipeline-UOSVI3Q5B4YDEQCAWIX3ITMD24.html sowie https://www.nabu.de/natur-undlandschaft/meere/lebensraum-meer/gefahren/23740.html

\section{CHRONIK}

\section{Covid-19-Chronik, 01. - 13. November 2021}

\begin{tabular}{|c|c|}
\hline 01.11 .2021 & $\begin{array}{l}\text { Nach Angaben des Moskauer Bürgermeisters Sergej Sobjanin wurden in der vergangenen Woche rund } 10.000 \\
\text { an Covid-19 erkrankte Personen in Moskauer Krankenhäuser eingeliefert. In den vergangenen } 24 \text { Stunden } \\
\text { wurden } 7.103 \text { Infektionsfälle in der Hauptstadt gezählt. 62,7 Prozent der Einwohner Moskaus sind bereits } \\
\text { vollständig geimpft. }\end{array}$ \\
\hline 03.11 .2021 & $\begin{array}{l}\text { In der Region Chabarowsk werden weitere Maßnahmen zur Eindämmung der Covid-19-Infektionszahlen erlas- } \\
\text { sen. Gastronomiebetriebe dürfen nur noch Essen zum Mitnehmen anbieten. Außerdem dürfen Einkaufszen- } \\
\text { tren, Hotels, Friseursalons, Schwimmbäder und andere Freizeiteinrichtungen nur gegen Vorlage eines Impf- } \\
\text { nachweises oder des Nachweises der Genesung von Covid-19 betreten werden. }\end{array}$ \\
\hline 03.11 .2021 & $\begin{array}{l}\text { Das russische Verteidigungsministerium teilt mit, dass die erste Einheit von mehr als } 220 \text { Soldaten zur Ent- } \\
\text { sendung in die Oblast Moskau zur Unterstützung medizinischer Einrichtungen in der Versorgung von Covid- } \\
\text { 19-Patienten bereit ist. Die Soldaten werden in einem Behelfskrankenhaus in Kubinka zur Unterstützung des } \\
\text { dortigen medizinischen Personals tätig werden. }\end{array}$ \\
\hline 03.11 .2 & $\begin{array}{l}\text { In Russland sterben innerhalb von } 24 \text { Stunden } 1.189 \text { Patienten an den Folgen einer Covid-19-Erkrankung. } \\
\text { Dies sind so viele wie nie seit Beginn der Pandemie im März } 2020 .\end{array}$ \\
\hline 03.11 .2021 & $\begin{array}{l}\text { In der Oblast Tscheljabinsk werden die zur Eindämmung des Coronavirus angeordneten arbeitsfreien Tage bis } \\
\text { einschließlich } 12 \text {. November } 2021 \text { verlängert. Ursprünglich sollte diese Regelung nur vom 30. Oktober bis zum } \\
\text { 07. November } 2021 \text { gelten. Die Oblast Nowgorod, die Oblast Smolensk sowie die Oblast Tomsk verlängerte } \\
\text { ebenfalls die arbeitsfreie Zeit. Diese Maßnahmen werden mit hohen Infektionszahlen begründet. }\end{array}$ \\
\hline 03.11 & $\begin{array}{l}\text { In der Oblast Uljanowsk wird gegen Mitarbeiter eines Krankenhauses ermittelt, die gegen Zahlung einer Gebühr } \\
\text { von } 2.000 \text { bis } 3.000 \text { Rubel (etwa } 24 \text { bis } 36 \text { Euro) gefälschte Impfdaten in offizielle Dokumente und die staat- } \\
\text { liche Datenbank eingetragen haben sollen. Auf diese Weise soll bisher } 500 \text { Personen eine gefälschte Impfbe- } \\
\text { scheinigung ausgestellt worden sein. }\end{array}$ \\
\hline 03.11 .2021 & $\begin{array}{l}\text { Der Gouverneur der Region Krasnodar, Wenjamin Kondratjew, erlässt ein Dekret, wonach ab sofort beim } \\
\text { Besuch gastronomischer Betriebe der Nachweis einer Impfung gegen Covid-19 oder der Genesung von der } \\
\text { Krankheit vorgelegt werden muss. Außerdem wird mit dem Dekret das Verbot der Durchführung von Veran- } \\
\text { staltungen bis zum } 14 \text {. November } 2021 \text { verlängert. Die Maßnahme wird mit den steigenden Covid-19-Infek- } \\
\text { tionszahlen begründet. }\end{array}$ \\
\hline
\end{tabular}




\begin{tabular}{|c|c|}
\hline 03.11 .2021 & $\begin{array}{l}\text { Die Leiterin der russischen Verbraucherschutzbehörde „Rospotrebnadsor«, Anna Popowa, verlängert die wegen } \\
\text { der Covid-19-Pandemie verhängten Einschränkungen für Veranstaltungen an Schulen und anderen Kinder- } \\
\text { und Jugendorganisationen bis zum 01. Januar 2024. Insbesondere wird das Verbot von Massenveranstaltungen } \\
\text { in geschlossenen Räumen ausgeweitet. Dies gilt nicht nur in Schulen oder speziellen Einrichtungen für Min- } \\
\text { derjährige, sondern zum Beispiel auch an Flughäfen, in Einkaufszentren oder an Bahnhöfen. Eine Ausnahme } \\
\text { gilt für berufsbildende Schulen sowie mittlere und höhere Bildungseinrichtungen. }\end{array}$ \\
\hline 04.11 .2021 & $\begin{array}{l}\text { In Russland sterben innerhalb von } 24 \text { Stunden 1.195 Patienten an den Folgen einer Covid-19-Erkrankung. } \\
\text { Dies sind so viele wie nie seit Beginn der Pandemie im März } 2020 .\end{array}$ \\
\hline 04.11 .2021 & $\begin{array}{l}\text { Der Pressedienst des regionalen Gesundheitsministeriums der russischen Teilrepublik Nordossetien-Alanien } \\
\text { teilt mit, dass aus dem Alchonskaja-Bezirkskrankenhaus } 25 \text { Covid-19-Patienten in andere Häuser verlegt wer- } \\
\text { den, um Engpässen bei der Sauerstoffversorgung vorzubeugen. }\end{array}$ \\
\hline 06.11 & $\begin{array}{l}\text { Mit } 41.335 \text { neuen Covid-19-Infektionsfällen innerhalb von } 24 \text { Stunden wird in Russland erneut eine Rekord- } \\
\text { zahl seit Beginn der Pandemie im März } 2020 \text { gemeldet. Das letzte Mal wurde am 31. Oktober } 2021 \text { ein Höchst- } \\
\text { stand gemeldet. }\end{array}$ \\
\hline 09.11 .2021 & $\begin{array}{l}\text { In Russland sterben innerhalb von 24 Stunden 1.211 Patienten an den Folgen einer Covid-19-Erkrankung. } \\
\text { Dies sind so viele wie nie seit Beginn der Pandemie im März } 2020 .\end{array}$ \\
\hline 09.1 & $\begin{array}{l}\text { Der russische Gesundheitsminister Michail Muraschko teilt mit, dass in Russland bisher } 62 \text { Millionen Men- } \\
\text { schen das erste Mal gegen Covid-19 geimpft seien. Dies entspricht rund } 43 \text { Prozent der gesamten Bevölkerung } \\
\text { und } 53 \text { Prozent der erwachsenen Bevölkerung. }\end{array}$ \\
\hline 10.11 .2021 & $\begin{array}{l}\text { Innerhalb von } 24 \text { Stunden erliegen in Russland } 1.239 \text { Menschen einer Covid-19-Erkrankung. Dies ist zum } \\
\text { zweiten Mal in Folge die höchste Zahl seit Beginn der Pandemie im März 2020. Die Sterblichkeit von Patien- } \\
\text { ten mit Covid-19 liegt damit bei vier Prozent. Patienten, die zur Behandlung an ein Beatmungsgerät ange- } \\
\text { schlossen werden, sterben in } 70 \text { bis } 95 \text { Prozent der Fälle. Dies teilt das russische Gesundheitsministerium mit. }\end{array}$ \\
\hline 10.11.2021 & $\begin{array}{l}\text { Die stellvertretende russische Ministerpräsidentin Tatjana Golikowa teilt mit, dass am Morgen des 09. Novem- } \\
\text { ber } 2021 \text { rund } 83 \% \text { der zur Behandlung von Covid-19-Patienten zur Verfügung stehenden Betten in der Rus- } \\
\text { sischen Föderation belegt waren. Insgesamt gäbe es } 301.500 \text { Betten, von denen an } 201.000 \text { Beatmungsgeräte } \\
\text { zur Verfügung stünden. Am } 20.10 .2021 \text { lag die Belegung nach Meldungen von Golikowa bei } 87 \% \text {. Zwischen- } \\
\text { zeitlich wurde neue Betten für Covid-Erkrankte geschaffen, Abteilungen und Krankenhäuser auf die Behand- } \\
\text { lung von Covid-Patienten umgestellt. }\end{array}$ \\
\hline 12.11 .2021 & $\begin{array}{l}\text { In der Region Kemerowo wird eine Pflicht zur Impfung gegen Covid-19 für Menschen ab } 60 \text { Jahren angeord- } \\
\text { net. In jüngster Zeit hatte es einen deutlichen Anstieg an Covid-19-Erkrankungen in der Altersgruppe der über } \\
\text { 60-Jährigen gegeben. Seit Juli } 2021 \text { gilt bereits eine Impfpflicht für Angehörige bestimmter Berufsgruppen. } \\
\text { Wenige Tage zuvor hatte auch St. Petersburg eine Impfpflicht für 60-Jährige und chronisch Kranke erlassen. }\end{array}$ \\
\hline 13.11 .2021 & $\begin{array}{l}\text { Russland liefert im Rahmen humanitärer Hilfe } 200.000 \text { Dosen des Codiv-19-Vakzins »Sputnik light« nach Kir- } \\
\text { gisistan. Das Vakzin wurde für den Einsatz in Hochinzidenzgebieten entwickelt. Seine Wirkung tritt bereits } \\
\text { nach einer Impfung ein, hält allerdings nur etwa sechs Monate an. }\end{array}$ \\
\hline 13.11.2021 & $\begin{array}{l}\text { Russland verzeichnet erneut einen Negativrekord bezüglich der Todesfälle im Zusammenhang mit einer Covid- } \\
\text { 19-Erkrankung. Innerhalb von } 24 \text { Stunden erlagen 1.241 Menschen der Erkrankung. }\end{array}$ \\
\hline
\end{tabular}

Zusammengestellt von Alena Schwarz

Die Covid-19-Chronik wird als Teil der klassischen Chronik mit Jahresbeginn 2022 fortgeführt und nicht mehr gesondert abgedruckt. Unsere Such- und Filterfunktionen der Webseite bieten die Möglichkeit innerhalb der Chronik mit den entsprechenden Stichworten "Corona" und "Covid" sowie einem Länder-Stichwort, sich alle Meldungen zu Covid-19 anzeigen zu lassen. Der Zeitraum im Suchverlauf kann eingegrenzt werden und die Stichworte "Corona" und "Covid sind gleichgesetzt, sodass auch die Treffer zum jeweils anderen Stichwort erscheinen. 


\section{1. - 12. November 2021}

\begin{tabular}{|c|c|}
\hline 01.11 .2021 & $\begin{array}{l}\text { Russland stellt die Zusammenarbeit mit der NATO ein. Den NATO-Mitarbeitern in Moskau wird ihre Akkre- } \\
\text { ditierung entzogen, gleichzeitig stellt die russische Vertretung bei der NATO in Brüssel ihre Tätigkeit ein. }\end{array}$ \\
\hline 02.11 .2021 & $\begin{array}{l}\text { Die Ukraine verhängt Sanktionen gegen die an den russischen Parlamentswahlen auf der Krim beteiligte Per- } \\
\text { sonen. Dazu gehören unter anderem Sergej Danilenko, Vorsitzender der regionalen Wahlkommission auf der } \\
\text { Krim, Jekaterina Pyrkowa, Leiterin der regionalen Abteilung des russischen Inlandsgeheimdienstes FSB auf } \\
\text { der Krim sowie Michail Raswoshajew, amtierender Gouverneur von Sewastopol. Außerdem wurden Sanktio- } \\
\text { nen gegen } 42 \text { russische Unternehmen verhängt. }\end{array}$ \\
\hline 02.11 .2021 & $\begin{array}{l}\text { In Moskau treffen sich der Direktor des russischen Auslandsgeheimdienstes (SWR), Sergej Naryschkin, und } \\
\text { der Direktor des US-amerikanischen Pendants CIA, William Burns. Das Treffen findet auf Initiative der USA } \\
\text { statt. Im Mittelpunkt steht die Frage nach der behördenübergreifenden Zusammenarbeit zur Bekämpfung des } \\
\text { internationalen Terrorismus. Burns traf in Moskau außerdem den Vorsitzenden des Sicherheitsrates der Rus- } \\
\text { sischen Föderation, Nikolaj Patruschew. }\end{array}$ \\
\hline 04.11 .2021 & $\begin{array}{l}\text { Der belarussische Präsident Aleksandr Lukaschenko unterzeichnet gemeinsam mit seinem russischen Amts- } \\
\text { kollegen Wladimir Putin ein Dekret, in dem er sich zu einem Unionsstaat mit Russland bekennt. Geplant ist } \\
\text { unter anderem eine engere militärische Zusammenarbeit sowie die Vereinheitlichung der Wirtschaftsgesetzge- } \\
\text { bung und der Renten- und Steuersysteme. Außerdem ist ein gemeinsamer Gas- und Ölmarkt geplant. }\end{array}$ \\
\hline 05.11 .2021 & $\begin{array}{l}\text { Ein Sprecher des Auswärtigen Amtes bestätigt Berichte des Nachrichtenmagazins "Der Spiegel«, wonach am } \\
\text { 19. Oktober } 2021 \text { ein Mitarbeiter der russischen Botschaft in Berlin tot aufgefunden worden sei. Offiziellen } \\
\text { Angaben zufolge soll der 35-Jährige aus einem Fenster der Botschaft gestürzt sein. Er war seit } 2019 \text { als zwei- } \\
\text { ter Sekretär der Botschaft akkreditiert. Nach Recherchen des "Spiegel" soll der Tote jedoch für den russischen } \\
\text { Inlandsgeheimdienst FSB gearbeitet haben. Die russischen Behörden bestreiten dies. }\end{array}$ \\
\hline 09.11 .2021 & $\begin{array}{l}\text { Der Vorsitzender der Moskauer Wahlkommission, Jurij Jermolow, tritt zurück. Sein Rücktritt habe persönli- } \\
\text { che Gründe, er bleibe einfaches Mitglied der Kommission, teilte die Leiterin der Zentralen Wahlkommission, } \\
\text { Ella Pamfilowa, mit. }\end{array}$ \\
\hline 09.11 .2021 & $\begin{array}{l}\text { Das Moskauer Stadtgericht nimmt den seit Mitte Oktober unter Hausarrest stehenden Rektor der Moskauer } \\
\text { Hochschule für Sozial- und Wirtschaftswissenschaften (»Schaninka»), Sergej Sujew, in Untersuchungshaft. } \\
\text { Gegen ihn wird wegen des Verdachts auf Betrug ermittelt. }\end{array}$ \\
\hline 11.11 .2021 & $\begin{array}{l}\text { Die russische Generalstaatsanwaltschaft beantragt beim Obersten Gerichtshof die Auflösung der russischen } \\
\text { Menschenrechtsorganisation »Memorial«. Eine Entscheidung wird am } 25 . \text { November } 2021 \text { fallen. Die Staats- } \\
\text { anwaltschaft wirft »Memorial« wiederholte Verletzung gegen das sogenannte »ausländische Agenten Gesetz« } \\
\text { vor, das Organisationen, die als ausländische Agenten geführt werden, vorschreibt, dies auf jeder ihrer Publi- } \\
\text { kationen zu kennzeichnen. }\end{array}$ \\
\hline 11.11 .2021 & $\begin{array}{l}\text { Zum zweiten Mal innerhalb von zwei Tagen führen der russische Präsident Wladimir Putin und die deutsche } \\
\text { Bundeskanzlerin Angela Merkel ein Telefongespräch zur Lage an den Grenzen zwischen Belarus und der Euro- } \\
\text { päischen Union. Seit Juli } 2021 \text { gelangen regelmäßig Flüchtlinge aus dem Irak, Syrien oder Afghanistan von } \\
\text { der belarussischen Grenzpolizei ungehindert über die Grenze nach Litauen und Polen. Merkel erhofft sich ein } \\
\text { Einwirken Putins auf Lukaschenko. Putin wiederum rät zu einer Wiederaufnahme der Kommunikation der } \\
\text { EU mit Belarus zur Lösung des Problems. }\end{array}$ \\
\hline 12.11 .2021 & $\begin{array}{l}\text { Der russische Außenminister Sergej Lawrow nimmt in Paris an einer internationalen Konferenz zur Lage in } \\
\text { Libyen teil. Außerdem wird er gemeinsam mit dem russischen Verteidigungsminister Sergej Schojgu zu einem } \\
\text { Acht-Augen-Gespräche mit dem französischen Außen- und dem Verteidigungsminister zusammenkommen. } \\
\text { Geplant ist neben Fragen der Rüstungskontrolle auch ein Meinungsaustausch zu aktuellen regionalen Themen } \\
\text { wie der Situation in der Ukraine, in Afghanistan oder im Iran. }\end{array}$ \\
\hline 12.11 .2021 & $\begin{array}{l}\text { Das russische Innenministerium setzt Wladimir Ossetschkin, Gründer des Menschenrechtsprojektes "Gulagu. } \\
\text { net« auf die Fahndungsliste. Ossetschkin war im Juli } 2020 \text { wegen Betrugs angeklagt worden; er hatte Russ- } \\
\text { land bereits } 2015 \text { verlassen und lebt zurzeit in Frankreich. Seit Juli } 2020 \text { wird er bereits über Interpol gesucht. } \\
\text { Vor einigen Wochen hatte er in Zusammenarbeit mit dem ehemaligen Häftling Sergej Saweljew ein Videoar- } \\
\text { chiv mit mehr als } 1.000 \text { Dateien veröffentlicht, das systematische Folter in russischen Gefängnissen aufdeckt. } \\
\text { Auch Saweljew hat inzwischen politisches Asyl in Frankreich beantragt. Die Seite »Gulagu.net« ist in Russ- } \\
\text { land seit Ende Juli } 2021 \text { blockiert. }\end{array}$ \\
\hline
\end{tabular}




\begin{tabular}{|l|l|}
\hline 12.11.2021 & $\begin{array}{l}\text { Der russische Regisseur Kirill Serebrennikow zahlt dem russischen Kulturministerium Schadensersatz in Höhe } \\
\text { von rund 129 Millionen Rubel (etwa 1,5 Millionen Euro). Außerdem begleicht er die ihm auferlegte Geldstrafe } \\
\text { in Höhe von 800.000 Rubel (etwa 9.500 Euro) vollständig. Serebrennikow war im Juni } 2020 \text { zusammen mit } \\
\text { zwei anderen Kulturschaffenden im sogenannten Fall »Sedmaja Studija« wegen Veruntreuung staatlicher Sub- } \\
\text { ventionen in derselben Höhe zu Geld- und Bewährungsstrafe verurteilt worden. Die gezahlte Summe stammt } \\
\text { nach Angaben Serebrennikows aus Spenden. Die Spender nannte er nicht. }\end{array}$ \\
\hline
\end{tabular}

Die Chronik wird zeitnah erstellt und basiert ausschließlich auf im Internet frei zugänglichen Quellen. Die Redaktion der RusslandAnalysen kann keine Gewähr für die Richtigkeit der Angaben übernehmen.

Zusammengestellt von Alena Schwarz

Sie können die gesamte Chronik seit 2003 (zusätzlich gibt es eine Kurzchronik für die Sowjetunion ab 1964 bzw. Russland ab 1992) auch auf http://www.laender-analysen.de/russland/ unter dem Link»Chronik« lesen.

Herausgeber:

Forschungsstelle Osteuropa an der Universität Bremen

Deutsche Gesellschaft für Osteuropakunde e.V.

Deutsches Polen-Institut

Leibniz-Institut für Agrarentwicklung in Transformationsökonomien

Leibniz-Institut für Ost- und Südosteuropaforschung

Zentrum für Osteuropa- und internationale Studien (ZOiS) gGmbH

Redaktion:

Anastasia Stoll (verantwortlich)

Chronik: Alena Schwarz

Satz: Matthias Neumann

Wissenschaftlicher Beirat:

Dr. Sabine Fischer, Stiftung Wissenschaft und Politik, Berlin

PD Dr. habil. Linde Götz, Leibniz-Institut für Agrarentwicklung in Transformationsökonomien

Prof. Dr. Alexander Libman, Freie Universität Berlin

Prof. Dr. Jeronim Perović, Universität Zürich

Dr. Cindy Wittke, Leibniz-Institut für Ost- und Südosteuropaforschung Regensburg

Die Meinungen, die in den Russland-Analysen geäußert werden, geben ausschließlich die Auffassung der Autoren wieder.

Abdruck und sonstige publizistische Nutzung sind nach Rücksprache mit der Redaktion gestattet.

Russland-Analysen-Layout: Cengiz Kibaroglu, Matthias Neumann und Michael Clemens

Alle Ausgaben der Russland-Analysen sind mit Themen- und Autorenindex archiviert unter www.laender-analysen.de

Die Russland-Analysen werden im Rahmen eines Lizenzvertrages in das Internetangebot der Bundeszentrale für politische Bildung (www.bpb.de) aufgenommen.

ISSN 1613-3390 @ 2021 by Forschungsstelle Osteuropa an der Universität Bremen

Forschungsstelle Osteuropa • Länder-Analysen • Klagenfurter Str. 8 • 28359 Bremen • Telefon: +49 421-218-69600 • Telefax: + 49 421-218-69607

e-mail: laender-analysen@uni-bremen.de•Internet-Adresse: http://www.laender-analysen.de/russland/ 
LÄNDER-ANALYSEN

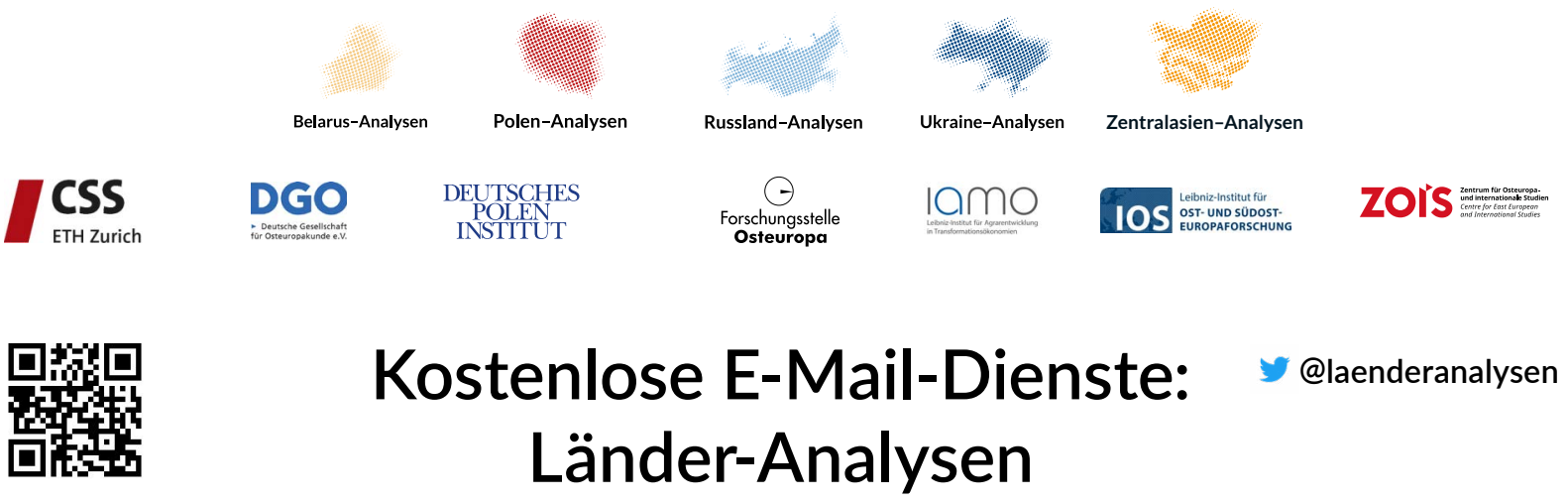

Die Länder-Analysen bieten regelmäßig im kostenlosen Abonnement kompetente Einschätzungen aktueller politischer, wirtschaftlicher, sozialer und kultureller Entwicklungen in Ostmitteleuropa und der GUS. Alle Länder-Analysen verstehen sich als Teil eines gemeinsamen Projektes, das der wissenschaftlich fundierten, allgemeinverständlich formulierten Analyse der Entwicklungen im östlichen Europa, der Offenheit für verschiedene inhaltliche Positionen und der kostenlosen und nicht-kommerziellen Information einer breit verstandenen interessierten Öffentlichkeit verpflichtet ist. Autor/innen sind internationale Fachwissenschaftler/innen und Expert/innen. Die Redaktionen der Länder-Analysen bestehen aus Wissenschaftler/innen mit langjähriger Forschungserfahrung.

Die deutschsprachigen Länder-Analysen werden gemeinsam von der Forschungsstelle Osteuropa an der Universität Bremen, dem Zentrum für Osteuropa- und internationale Studien, der Deutschen Gesellschaft für Osteuropakunde, dem Deutschen Polen-Institut, dem Leibniz-Institut für Agrarentwicklung in Transformationsökonomien und dem Leibniz-Institut für Ost- und Südosteuropaforschung herausgegeben. Die englischsprachigen Länder-Analysen erscheinen in Kooperation der Forschungsstelle Osteuropa mit dem Center for Security Studies (CSS) der ETH Zürich.

Die Länder-Analysen bieten regelmäßig Kurzanalysen zu aktuellen Themen, ergänzt um Grafiken und Tabellen sowie Dokumentationen. Zusätzlich gibt es eine Chronik aktueller Ereignisse.

\section{Belarus-Analysen}

Erscheinungsweise: zweimonatlich

Abonnement unter: http://www.laender-analysen.de/belarus/

\section{Caucasus Analytical Digest}

In englischer Sprache. Erscheinungsweise: zweimonatlich

Abonnement unter: http://www.css.ethz.ch/en/publications/cad.html

\section{Polen-Analysen}

Erscheinungsweise: zweimal monatlich

Abonnement unter: http://www.deutsches-polen-institut.de/newsletter/polen-analysen/

\section{Russland-Analysen}

Erscheinungsweise: zweimal monatlich

Abonnement unter: http://www.laender-analysen.de/russland/

\section{Russian Analytical Digest}

In englischer Sprache. Erscheinungsweise: zweimal monatlich Abonnement unter: http://www.css.ethz.ch/en/publications/rad.html

\section{Ukraine-Analysen}

Erscheinungsweise: zweimal monatlich

Abonnement unter: http://www.laender-analysen.de/ukraine/

\section{Zentralasien-Analysen}

Erscheinungsweise: zweimonatlich

Abonnement unter: http://www.laender-analysen.de/zentralasien/ 